\title{
Epidermoid Cyst of the Spleen in the Laparoscopic Era
}

\author{
Isidoro Di Carlo Maria Antonietta Fasone Adriana Toro \\ Department of Surgical Sciences, Organs Transplantation and Advanced Technologies, University of Catania, \\ Cannizzaro Hospital, Catania, Italy
}

L.P.A., a 13-year-old girl, presented with left upper abdominal pain due to an accidental fall. Ultrasonography (US) showed a cystic mass which appeared to be a hematoma, but the patient was hemodynamically stable. Spiral computer tomography (CT) showed a 20 -cm cystic mass of the spleen occupying almost all the splenic parenchyma (fig. 1). The cyst was septed with smooth wall and fluid content (fig. 2). This finding excluded the diagnosis of a hematoma and suggested the hypothesis of hydatidosis or congenital cyst of the spleen. Echinococcosis antibodies were negative so a definite diagnosis could not be made and surgical treatment was required. The splenectomy was performed by laparotomy. The spleen weighed $1,080 \mathrm{~kg}$ and the fluid inside it was turbid and yellow in

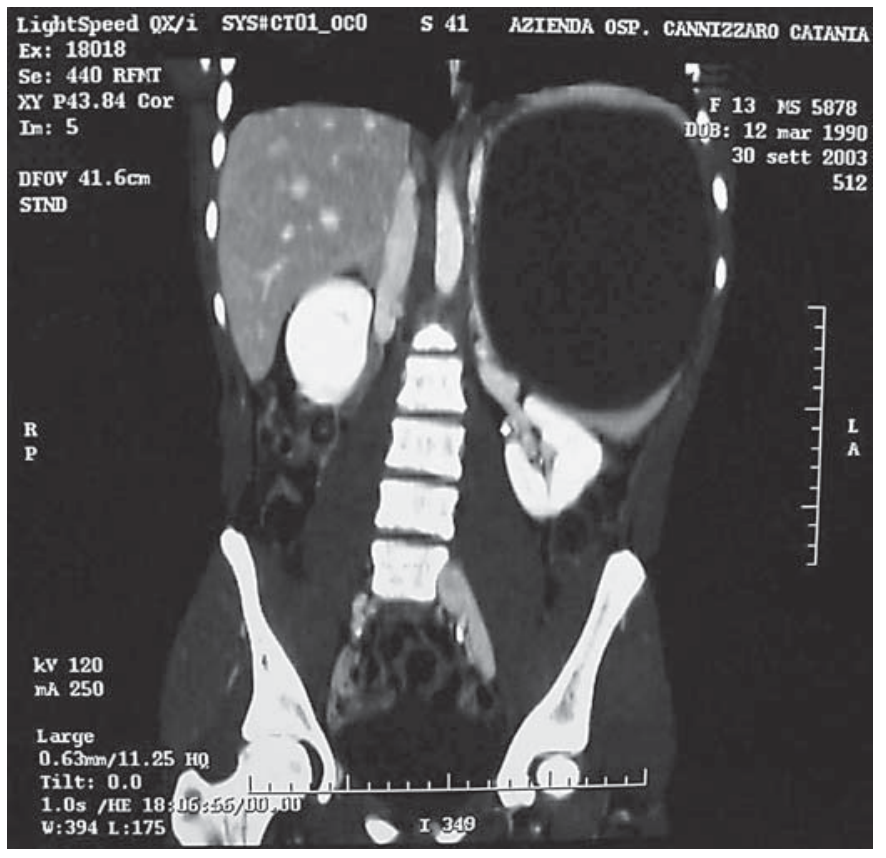

Fig. 1. Spiral CT sagittal view of the giant cyst replacing almost all of the splenic parenchyma.

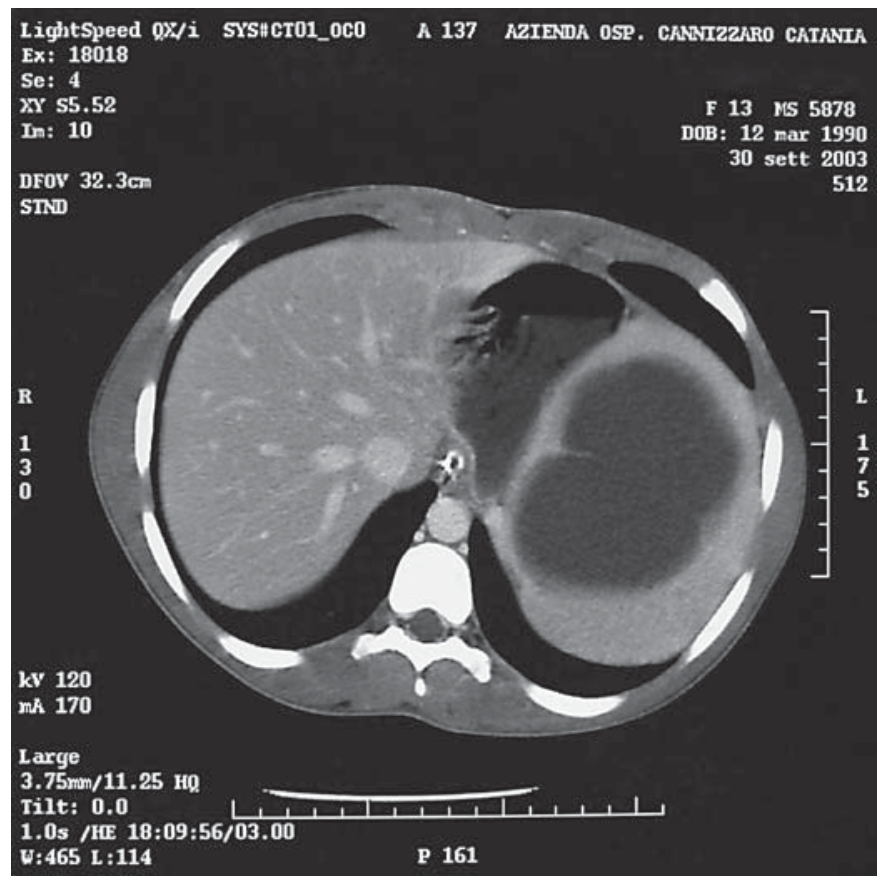

Fig. 2. Spiral CT view of the septated wall of the mass.

Isidoro Di Carlo, $\mathrm{MD}, \mathrm{PhD}$

Department of Surgical Sciences, Organ Transplantation and Advanced Technologies

University of Catania, c/o Cannizzaro Hospital, Via Messina, 829

IT-95126 Catania (Italy)

Tel./Fax +39095 7263020, E-Mail idicarlo@unict.it 
color (fig. 3). Pathological examination showed an epidermoid congenital cyst with fibrous and calcified wall; the remaining spleen parenchyma was atrophic (fig. 4). The postoperative course was free of complications and the patient was discharged from the hospital 7 days after surgery.
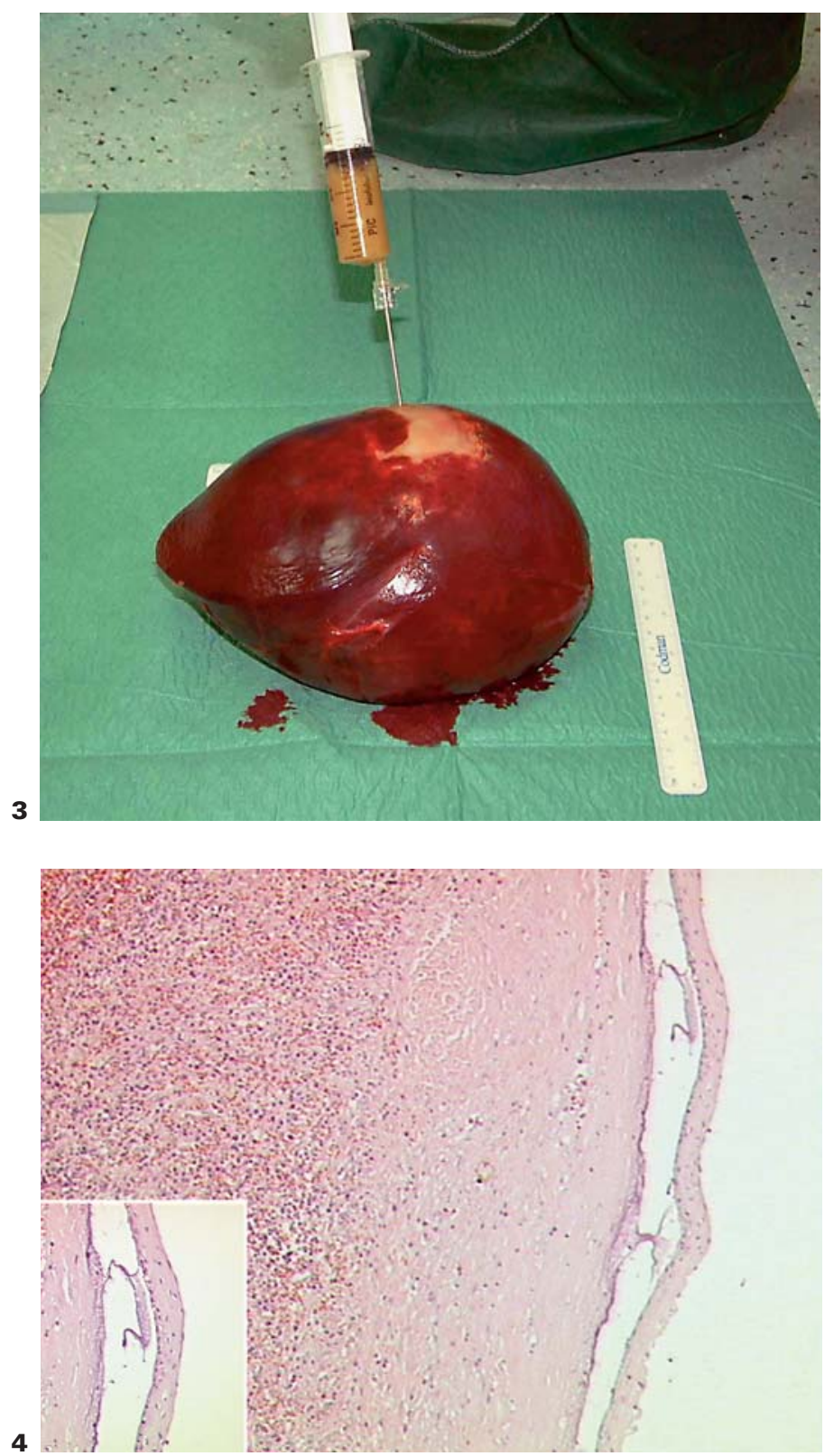

\section{Discussion}

Congenital epidermoid splenic cysts are rare and only about 1,000 cases have been reported in the literature [1]. The cystic fluid can be thin and serous but cysts with turbid and viscid fluid have been reported as well. Cyst presents as an asymptomatic abdominal mass or with pain in the left upper quadrant due to the enlargement, hemorrhage, rupture or infection [2]. Even if US is the first examination to evaluate the spleen, CT scan is more useful because it can clearly establish size, position, and type of fluid content; however, the CT examination could not be diagnostic for the nature of the cyst. Conventional treatment of nonparasitic cyst is total splenectomy, but alternative treatment options including aspiration, internal and external marsupialization, partial splenectomy and partial cystectomy (decapsulation) have been reported. These treatments can be performed by open or laparoscopic surgery [2]. Nonoperative treatment is recommended for small cysts, up to $5 \mathrm{~cm}$ in diameter, if the cysts are totally asymptomatic and the imaging characteristics are absolutely typical of nonparasitic splenic cysts. Laparoscopic surgery provides a minimal access method to approach small true cysts of the liver and the spleen, but in our patient the cyst was very large, centrally located and the splenic parenchyma was thin as it was almost completely occupied by the mass. Moreover, the preoperative diagnosis was uncertain and for the young age and the risk of eventual dissemination of cyst fluid, we choose to perform a laparotomy. Besides, complete removal of the cyst is needed because recurrence of epidermoid cysts has been reported both after open and laparoscopic surgery [3]. In addition, conservative treatment is not possible when the spleen is almost totally occupied by the cyst [4] as in our case in which we couldn't eradicate the cyst without performing a total splenectomy.

Fig. 3. The great volume of the spleen due to the cyst. A turbid liquid is aspirated.

Fig. 4. Histological findings of the epidermoid cyst showing red pulp of the splenic parenchyma on the left, and one fibrous cystic wall covered by stratified pavement epithelium on the right. $\times 4$. Inset: A segment of the pavement epithelium. $\times 10$.

\section{References}

Dig Surg 2005;22:53-54
1 Morgenstern L: Non parasitic splenic cysts: Pathogenesis, classification, and treatment. J Am Coll Surg 2002;194:306-314.

2 Seshadri PA, Park A, et al: Laparoscopic splenic cystectomy: A case report. J Pediatr Surg 1998;33:1439-1440.

3 Ganti AL, Sardi A, Gordon J: Laparoscopic treatment of large true cysts of the liver and the spleen is ineffective. Am Surg 2002;68:10121017.

4 Musy PA, Le Coultre C, et al: Splenic cysts in pediatric patients: A report on 8 cases and review of literature. Eur J Pediatric Surg 1992;2: 137-140.

Di Carlo/Fasone/Toro 